\title{
CAUCHY PROBLEM OF HYPERBOLIC CONSERVATION LAWS IN MULTIDIMENSIONAL SPACE WITH INTERSECTING JUMP INITIAL DATA
}

\author{
DENING LI
}

\begin{abstract}
Cauchy problem of hyperbolic conservation laws in multidimensional space is considered, where the initial data have several jump discontinuity surfaces which develop into shock fronts intersecting at a common submanifold. Local existence is proved, assuming compatible conditions and uniform stability. For isentropic flow in 2-dimensional space, the interaction of two shock fronts and the nonexistence of three intersecting shock fronts are discussed.
\end{abstract}

Introduction. In [1], the Cauchy problem was discussed for hyperbolic conservation laws in multidimensional space with initial data which have jump discontinuity on a smooth hypersurface and this initial jump develops into two or more shock fronts in $t>0$. In discussing the interaction of two shock fronts, because of the fact that at fixed time $t=t_{0}$, part of the bumping shock fronts remains unchanged while the other part has already produced new shock fronts, it is necessary to consider the Cauchy problem with initial data which have jump discontinuity on more than one hypersurfaces that intersect with each other.

In [7], Metivier considered the interaction of two shock fronts for 2 conservation laws in 2-dimensional space. He reduced the problem of interaction to the problem of double shock fronts emanating from one discontinuity surface. For the stability analysis, we can follow the similar approach to discuss the stability of interaction of shock fronts for $m$ conservation laws in $n$-dimensional space. But for the problem of existence, the situation in the general case becomes different from the one discussed in [7]. Certain additional conditions are necessary to get the existence result.

In this paper, the general $m$ conservation laws in $n$-dimensional space will be discussed, not only for the problem of interaction of shock fronts, but also for the problem of other features. In particular, we discuss the isentropic hydrodynamic equations in 2-dimensional space and get the interesting result of the interaction of two shock fronts and that it is always impossible to have three stable shock fronts intersecting at one common curve.

1. Problem and result. As in $[\mathbf{1}, \mathbf{4}, \mathbf{5}]$, we discuss the following hyperbolic conservation laws

$$
D_{t}\left(F_{0}(u)\right)+\sum_{1}^{n} D_{x_{j}}\left(F_{j}(u)\right)=0
$$

Received by the editors January 26, 1987 and, in revised form, April 27, 1987. $76 \mathrm{~L} 05$.

1980 Mathematics Subject Classification (1985 Revision). Primary 35L50, 35L65; Secondary

Supported by NSERC (Canada). 


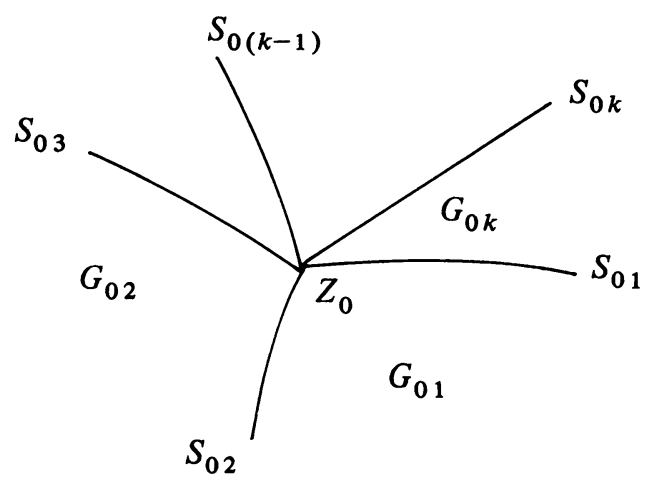

FIGURE 1

which can be written as a quasilinear symmetric hyperbolic system

$$
D_{t} u+\sum_{1}^{n} A_{j}(u) D_{x_{j}} u+B(u) u=F(x, t) .
$$

Besides, we always assume that the linearization of (1.2) satisfies the block structural condition in [4].

We consider the Cauchy problem of (1.1) with the initial data

$$
u(x, 0)=u_{0}(x) .
$$

Here $u_{0}(x)$ is a piecewise smooth function having discontinuities of the first kind on $k$ smooth hypersurfaces $S_{0 i}(i=1, \ldots, k)$, which intersect transversally at a submanifold $Z_{0}$ of dimension $(n-2)$; see Figure 1 .

Denote the domain between $S_{0 i}$ and $S_{0(i+1)}$ by $G_{0 i}(i=1, \ldots, k)$, where $S_{0(k+1)}$ $=S_{01}$. We will always assume in the following that every $G_{0 i}$ is diffeomorphic to a quarter space in $R^{n}$. Denote the value of $u_{0}(x)$ in $G_{0 i}$ by $u_{0 i}(x)$. We assume $u_{0 i}(x) \in C^{\infty}\left(\overline{G_{0 i}}\right)$ and $u_{0 i}(x) \neq u_{0(i-1)}$ on $S_{0 i}$, for $i=2, \ldots, k+1$.

In this paper, we are going to prove that, under certain assumptions on $u_{0 i}$ $(i=1, \ldots, k)$, there exists a $t_{0}>0$, such that for $0<t<t_{0}$, the Cauchy problem (1.2), (1.3) has piecewise smooth solution $u(x, t)$ with discontinuity surfaces $S_{i}$ $(i=1, \ldots, k)$ which intersect each other on a common submanifold $Z$ of dimension $(n-1)$ in $R^{n} \times R_{+}^{1}$ and $\left.S_{i}\right|_{t=0}=S_{0 i},\left.Z\right|_{t=0}=Z_{0}$. And on each $S_{i}, u$ has a shock wave discontinuity, satisfying Rankine-Hugoniot conditions

$$
D_{t} p_{i}\left[F_{0}(u)\right]^{i}+\sum_{1}^{n} D_{x_{j}} p_{i}\left[F_{j}(u)\right]^{i}=0, \quad \text { on } S_{i}, i=1, \ldots, k .
$$

Here $[f]^{i}$ denotes the jump difference of $f$ across $S_{i}$, and $S_{i}=\left\{(x, t) ; p_{i}(x, t)=0\right\}$.

Denote the unit normal vector on $S_{0 i}$ by $\vec{n}_{i}=\left(n_{i 1}, \ldots, n_{i n}\right), i=1, \ldots, k$. We make the following assumptions:

On every $S_{0 i}$, there exists a scalar function $n_{i 0}$, sufficiently smooth on $S_{0 i}$, such that

$$
n_{i 0}\left[F_{0}(u)\right]^{i}+\sum_{1}^{n} n_{i j}\left[F_{j}(u)\right]^{i}=0, \quad \text { on } S_{0 i}, i=1, \ldots, k .
$$


When (H1) is satisfied, then by changing the sign of $\left(n_{i 1}, \ldots, n_{i n}\right)$ if necessary, we may always take $n_{i 0} \geq 0$.

$$
\begin{aligned}
& \text { On every } S_{0 i}, \text { the vector }\left(n_{i 0}, n_{i 1}, \ldots, n_{i n}\right) \text { and the state }\left(u_{0 i}, u_{0(i-1)}\right) \\
& \text { satisfy the uniform stability condition for shock front of Majda [4]. }
\end{aligned}
$$

At $Z_{0}, k$ vectors $\left(n_{i 0}, n_{i 1}, \ldots, n_{i n}\right)(i=1, \ldots, k)$ lie in a common hyperplane of dimension 2.

The assumption (H3) is in fact equivalent to the hypothesis that the hyperplanes with normal vector $\left(n_{i 0}, n_{i 1}, \ldots, n_{i n}\right)$ intersect at a common affine submanifold of dimension $(n-1)$, which will be denoted as $Z_{t}$ in the following.

Obviously, the assumptions (H1)-(H3) are necessary for the existence of the intersecting stable shock fronts.

Another assumption we are going to make is connected with the concept of extreme shock. As in the case of one dimensional space, every shock front is associated to one genuinely nonlinear characteristic of the system (1.2), cf. e.g. J. Smoller [8]. We are here interested only in the extreme shock front which is associated to an extreme characteristic, i.e., the characteristic of the largest or smallest eigenvalue. In this situation, the direction $\left(n_{i 0}, n_{i 1}, \ldots, n_{i n}\right)$ is space-like with respect to the value $u_{0}$ in one and only one side of $S_{i 0}$. And consequently, we get $n_{i 0}>0$. The following is our fourth assumption

$$
\begin{aligned}
& \text { There is a domain } G_{0 i} \text {, such that }\left(n_{i 0}, n_{i 1}, \ldots, n_{i n}\right) \text { and }\left(n_{(i+1) 0}\right. \text {, } \\
& \left.n_{(i+1) 1}, \ldots, n_{(i+1) n}\right) \text { are both space-like with respect to } u_{i 0} ; \text { and } \\
& \text { these two vectors are both pointing outward from } G_{0 i} \text {. }
\end{aligned}
$$

REMARK. The second statement in (H4) is equivalent to the requirement that the intersection $Z_{t}$ of the hyperplanes with normals $\left(n_{i 0}, n_{i 1}, \ldots, n_{i n}\right)$ and $\left(n_{(i+1) 0}\right.$, $\left.n_{(i+1) 1}, \ldots, n_{(i+1) n}\right)$ has its projection into the space of $x=\left(x_{1}, \ldots, x_{n}\right)$ contained in the domain $G_{0 i}$.

In particular, for Euler equations in gas dynamics, we could have at most two shock fronts developing from one discontinuity, and these two shock fronts are associated with the largest and the smallest eigenvalues respectively [4]. Since the uniformly stable shock front in gas dynamics with convex state function must be compressive, i.e., the pressure of the gas behind the shock front should be higher than the pressure ahead of the shock front, it is easy to see that there is always one and only one domain $G_{i 0}$ such that the pressures in the adjacent domains are higher than the one in $G_{i 0}$. So for Euler equations of gas dynamics, hypothesis (H4) is automatically satisfied.

To fix the idea, we will always denote the domain in (H4) as $G_{01}$.

To have a piecewise sufficiently smooth solution with smooth discontinuity surface, it is necessary to have compatibility conditions which are derived from equation (1.1) and Rankine-Hugoniot conditions (1.4). Hence, we make the following assumption:

On every $S_{0 i}$, the compatibility conditions are satisfied up to sufficiently high order.

REMARK. (H1) is nothing but the compatibility condition of order zero. The high order compatibility conditions are the requirements upon $u_{0}$ to guarantee the existence of compatible $D_{t}^{h} p_{i}, i=1, \ldots, k ; h=1, \ldots, L$, for sufficiently large $L$. 
In order to get the solution with similar discontinuity picture as Figure 1 in $t>0$, we also need the higher order forms of (H3):

There exists a submanifold $Z$ of dimension $(n-1)$ containing $Z_{0}$ such that $Z$ is tangent up to high order at $Z_{0}$ to every hypersurface $S_{i}$ which passes through $S_{0 i}$ and has the same higher order curvatures at $Z_{0}$ determined by $D_{t}^{h} p_{i}, i=1, \ldots, k ; h=1, \ldots, L$.

In particular, if the initial discontinuity $S_{0 i}$ 's are all hyperplanes and $u_{0}$ in every $G_{0 i}$ is constant state, then (H6) is automatically satisfied.

Now, we are going to make our last assumption. Since in $G_{01}$, two directions $\left(n_{10}, n_{11}, \ldots, n_{1 n}\right)$ and $\left(n_{20}, n_{21}, \ldots, n_{2 n}\right)$ are both space-like with respect to $u_{0}$, so by the uniform stability assumption $(\mathrm{H} 2),\left(n_{10}, \ldots, n_{1 n}\right)$ and $\left(n_{20}, \ldots, n_{2 n}\right)$ could not be space-like with respect to $u_{0 k}$ and $u_{02}$, respectively. Examining the possibility in the adjacent domains $G_{02}$ and $G_{0 k}$, we have three different cases:

(1.6)(i) $\left(n_{30}, \ldots, n_{3 n}\right)$ and $\left(n_{k 0}, \ldots, n_{k n}\right)$ are not space-like with respect to $u_{02}$ and $u_{0 k}$, respectively;

(ii) $\left(n_{30}, \ldots, n_{3 n}\right)$ and $\left(n_{k 0}, \ldots, n_{k n}\right)$ are both space-like with respect to $u_{02}$ and $u_{0 k}$, respectively;

(iii) One of $\left(n_{30}, \ldots, n_{3 n}\right)$ and $\left(n_{k 0}, \ldots, n_{k n}\right)$ is space-like, another is not.

To fix the idea in case (iii), we will always take $\left(n_{k 0}, \ldots, n_{k n}\right)$ being space-like with respect to $u_{0 k}$, and $\left(n_{30}, \ldots, n_{3 n}\right)$ not space-like with respect to $u_{02}$.

REMARK. For the three cases cited above, the case (i) can never happen in gas dynamics, because the stable shock front must be associated with extreme characteristics and hence we have one and only one domain $G_{0 i}$ which has two non-space-like boundaries.

Now let $\tilde{G}_{0}$ be the subset of the domains $G_{0 i}$ :

$\tilde{G}_{0}=\left\{G_{0 i} ; i=3, \ldots, k-1\right.$ or $i=2, k$ and $G_{0 i}$ has two non-space-like boundaries $\}$. Let $q$ be the number of $G_{0 i}$ in the set $\tilde{G}_{0}$. We have $k-3 \leq q \leq k-1$.

On all $q+1$ boundaries which are adjacent to at least one domain $G_{0 i} \in \tilde{G}_{0}$, we have the Rankine-Hugoniot conditions

$$
D_{t} p_{i}\left[F_{0}\left(u_{i+1}\right)-F_{0}\left(u_{i}\right)\right]+\sum_{1}^{n} D_{x_{j}} p_{i}\left[F_{j}\left(u_{i+1}\right)-F_{j}\left(u_{i}\right)\right]=0, \quad \text { on } S_{i} \text {. }
$$

These are $m(q+1)$ relations. Besides, the $(q+1)(n+1)$ components of $\left(D_{t} p_{i}, D_{x} p_{i}\right)$ are not independent of each other. The normalizations

$$
\left|D_{t} p_{i}\right|^{2}+\sum_{1}^{n}\left|D_{x_{j}} p_{i}\right|^{2}=1, \quad G_{0 i} \in \tilde{G}_{0} \text { or } G_{0(i+1)} \in \tilde{G}_{0}
$$

are $(q+1)$ relations. And as in (H3), they should lie in a common hyperplane of dimension 2, i.e., there exist $(n-1)$ unit vectors $\vec{a}_{j}$ in $R^{n} \times R_{+}^{1}, i=1, \ldots,(n-1)$, such that

$$
\left(\vec{a}_{i}, \vec{a}_{j}\right)=\delta_{i j}, \quad i, j=1, \ldots,(n-1)
$$

and

$$
\left(\left(D_{t} p_{i}, D_{x} p_{i}\right), \vec{a}_{j}\right)=0, \quad j=1, \ldots,(n-1) ; G_{0 i} \text { or } G_{0(i+1)} \in \tilde{G}_{0}
$$


Once the intersecting submanifold $Z$ is given, then the $\vec{a}_{j}$ 's in (1.9), (1.10) are determined uniquely up to orthogonal transformations within themselves, while (1.10) is invariant for such transformations. Hence, we will have $(q+1)(n-1)$ relations for $\left(D_{t} p_{i}, D_{x} p_{i}\right)$ in $(1.10)$.

If $Z$ is partly given, i.e., if $Z$ is required to lie within a given surface $S_{2}$, then the $a_{j}$ 's in (1.10) should satisfy another $(n-1)$ supplementary conditions

$$
\left.\overrightarrow{(b}, \vec{a}_{j}\right)=0, \quad j=1, \ldots, n-1,
$$

where $\vec{b}$ is a given vector (the normal vector of $S_{2}$ ). Eliminating part of $a_{j}$ 's in $(1.10)$ by $(1.11)$, we get $(n-1) q$ relations for $\left(D_{t} p_{i}, D_{x} p_{i}\right)$, again denoted as $(1.10)$, when there is no confusion possible.

If $Z$ is completely not given, then we will have $(q-1)(n-1)$ relations in $(1.10)$ for $\left(D_{t} p_{i}, D_{x} p_{i}\right)$.

From the examples discussed in $\S \S 3$ and 4 , we will see that for $n=2$, the condition (1.10) is extremely simple. It consists of the determinant of a 3-order matrix and the introduction of $\vec{a}$ 's is not necessary.

Now, we will view $u_{i}$ in $G_{i}$ with $G_{0 i} \in \tilde{G}_{0}$ and $\left(D_{t} p_{i}, D_{x} p_{i}\right),\left(D_{t} p_{i+1}, D_{x} p_{i+1}\right)$ as unknowns. For these $q_{1}=m q+(q+1)(n+1)$ unknowns, denoted by $U$ in the following, we have $m(q+1)$ Rankine-Hugoniot conditions $(1.7)$ on $(q+1) S_{i}$, which is adjacent to at least one $G_{o i} \in \tilde{G}_{0}$. Besides, we have $(q+1)$ normalizing conditions (1.8) and certain number of conditions in (1.10) to determine the position of every $S_{i}$. The exact number of relations in (1.10) will depend on the determination state of $Z$.

Denote all the relations in (1.7), (1.8) and (1.10) as

$$
Q(U)=0 .
$$

Consider the three cases in (1.6).

In case (i), we have $q=k-1$. Since now all $(q+1) S_{i}$ 's are taken unknowns, $Z$ is not given beforehand. So we have in fact $(q-1)(n-1)$ relations in (1.10). Hence for $q_{1}$ unknowns $U$, we have $q_{2}=m(q-1)+(q+1)+(q-1)(n-1)=$ $(m+n)(q+1)-2(n-1)$ relations in $(1.12)$.

In case (ii), we have $q=k-3$. Now $Z$ is determined by $S_{1}$ and $S_{2}$, so we have $(q+1)(n-1)$ relations in $(1.10)$. Therefore, for $q_{1} U$, we have $q_{2}=m(q+1)+$ $(q+1)+(q+1)(n-1)=(m+n)(q+1)$ relations in (1.12).

In case (iii), $q=k-2$. Now $Z$ is required to be on a given surface $S_{1}$. So we have in fact $q(n-1)$ relations in (1.10). And for $q_{1}$ unknowns $U$, we have $q_{2}=m(q+1)+(q+1)+q(n-1)=(m+n)(q+1)-(n-1)$ relations in (1.12).

For all these three cases where $q_{2}$ takes different values, we impose the following requirement

Jacobian $D Q / D U$ has rank $q_{2}$ at $t=0$ and $x \in Z_{0}$.

COROLlaRY 1. From (H7), we get at once that $q_{2} \leq q_{1}$. In case (i), it means $q \geq m-2 n+1$. In case (ii), it means $q \geq m-1$. In case (iii), it means $q \geq m-n$.

REMARK 2. Since only one shock front could be associated with one genuinely nonlinear eigenvalue of the characteristic matrix of the system, we should always 
have $q+1 \leq m$. This is an absolute restriction on the number of initial discontinuities. In particular, in case (ii), this implies by Corollary 1 that $q=m-1$.

REMARK 3. Consider the particular problem of interaction of shock fronts in 2-dimensional space for two hyperbolic laws considered by Metivier in [7]. It corresponds to the case (ii) considered here, with $m=2, q=1$, exactly the only possible situation when (H7) can be satisfied.

Generally speaking, we cannot get rid of $(\mathrm{H} 7)$ in order to get our desired result. Nevertheless, it is only a sufficient condition. In some special cases, e.g., in the examples of $\S 3$, where the condition in Corollary 1 is not satisfied, we still get the existence result.

Now, the main result of this paper can be stated as follows.

THEOREM. Suppose that the Cauchy problem (1.1),(1.3) with intersecting discontinuity data satisfy all the conditions (H1)-(H7). Then there exists a $t_{0}>0$, such that (1.1),(1.3) has a piecewise differentiable solution $u$ in $0<t<t_{0}$, which has jump discontinuity at differentiable surfaces $S_{i}$ 's, intersecting with each other at a common submanifold $Z$ of dimension $(n-1)$, and on $S_{i}$, Rankine-Hugoniot conditions (1.4) are satisfied.

2. Proof of the Theorem. The basic idea of the proof consists of two steps. First, we try to determine the position of $Z$. Then, by a transformation, we reduce the given problem to a problem of multishock fronts we discussed in [1].

First of all, by (H4), we know that the $(n-1)$-dimensional hyperplane $Z_{t}$, perpendicular to $\left(n_{10}, n_{11}, \ldots, n_{1 n}\right)$ and $\left(n_{20}, n_{21}, \ldots, n_{2 n}\right)$, has its projection in $x$-space contained in $G_{01}$ for $t>0$. We now discuss the three cases in (1.6).

Case (i). In this case, for $q_{1}$ unknown variables $\left(D p_{1}, \ldots, D p_{k}\right)$ and $\left(u_{2}, \ldots, u_{k}\right)$, we have $q_{2}$ independent relations $Q(U)=0$. But at $t=0$, according to our assumptions (H1) and (H3), we know $U_{0}=\left(\vec{n}_{1}, \ldots, \vec{n}_{k}, u_{02}, \ldots, u_{0 k}\right)$ satisfies all these relations, i.e., $Q\left(U_{0}\right)=0$. Thus, from $(\mathrm{H} 7)$ and the implicit function theorem, we know that near $t=0, x \in Z_{0}$, there is a vector function $U(t, x)$ satisfying $Q(U(t, x))=0$ and $\left.U(0, x)\right|_{x \in Z_{0}}=U_{0}$. It is worth pointing out here that the function $U(t, x)$ is determined uniquely only when $q_{2}=q_{1}$. Otherwise $U(t, x)$ may not be unique. (Similar argument also applies to the discussion of cases (ii) and (iii).) Now $\left(D p_{1}, \ldots, D p_{k}\right)$ satisfies (1.10) near $t=0, x \in Z_{0}$, and it determines a submanifold $Z$ of dimension $(n-1)$, with $\left.Z\right|_{t=0}=Z_{0}$.

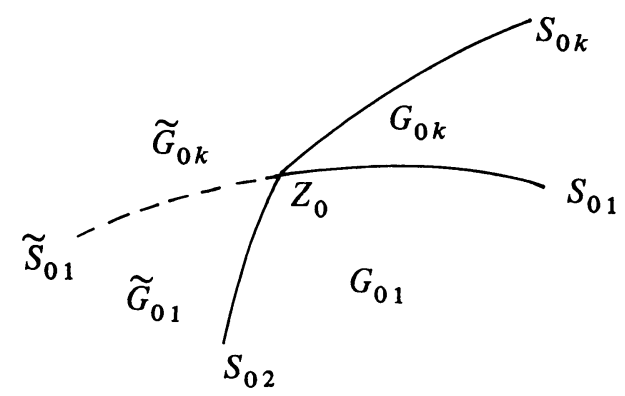

FIGURE 2 
Case (ii). In this case, $\left(n_{30}, \ldots, n_{3 n}\right)$ and $\left(n_{k 0}, \ldots, n_{k n}\right)$ are both space-like with respect to $u_{02}$ and $u_{0 k}$. We are now to follow the approach of G. Metivier. First, we extend the hypersurface $S_{01}$ beyond $Z_{0}$ into a smooth hypersurface $\tilde{S}_{01}$ without boundary. $\tilde{S}_{01}$ will divide $R^{n}$ into two new domains, denoted as $\tilde{G}_{01}$ and $\tilde{G}_{0 k}$, with $G_{01}$ contained in $\tilde{G}_{01}$ and $G_{0 k}$ in $\tilde{G}_{0 k}$. See Figure 2.

And also, we extend $u_{01}$ and $u_{0 k}$ smoothly into $\tilde{G}_{01}$ and $\tilde{G}_{0 k}$ such that the extended values $\tilde{u}_{01}$ and $\tilde{u}_{0 k}$ satisfy all Rankine-Hugoniot conditions and the uniform stability condition of Majda in [4]. By the result of Majda [5], we know the following Cauchy problem

$$
\left\{\begin{array}{l}
D_{t}\left(F_{0}(u)\right)+\sum_{1}^{n} D_{x_{j}}\left(F_{j}(u)\right)=0, \quad \text { in } t>0, x \in R^{n}, \\
u(x, 0)= \begin{cases}u_{01}(x), & x \in \tilde{G}_{01}, \\
u_{0 k}(x), & x \in \tilde{G}_{0 k},\end{cases}
\end{array}\right.
$$

has a local shock wave solution $u(x, t)$ which is sufficiently smooth on either side of a sufficiently smooth hypersurface $\tilde{S}_{1}$. Denote two domains separated by $\tilde{S}_{1}$ as $\tilde{G}_{1}$ and $\tilde{G}_{k}$, with $\tilde{G}_{01}$ contained in $\tilde{G}_{1}$, and $\tilde{G}_{0 k}$ in $\tilde{G}_{k}$. The value of $u(x, t)$ in $\tilde{G}_{1}$ and $\tilde{G}_{k}$ will be denoted as $\tilde{u}_{1}(x, t)$ and $\tilde{u}_{k}(x, t)$.

Similarly, we can extend hypersurface $S_{02}$ across $Z_{0}$ into $\hat{S}_{02}$ which separates $\hat{G}_{01}$ and $\hat{G}_{02}$. Also, we extend $u_{01}$ and $u_{02}$ into $\hat{u}_{01}$ and $\hat{u}_{02}$, and then solve the Cauchy problem with initial data $\left(\hat{u}_{01}, \hat{u}_{02}\right)$, having one jump discontinuity. The resulted shock wave solution will be denoted by $\hat{u}_{1}(x, t), \hat{u}_{2}(x, t)$ and $\hat{S}_{2}$ which separates $\hat{G}_{1}$ and $\hat{G}_{2}$.

Now in the domain $G_{1}=\tilde{G}_{1} \cap \hat{G}_{1}$, we have $\tilde{u}_{1}(x, t)=\hat{u}_{1}(x, t)$. The conclusion follows from the fact that the directions $\left(n_{10}, \ldots, n_{1 n}\right)$ and $\left(n_{20}, \ldots, n_{2 n}\right)$ are both space-like with respect to $u_{01}$. At $t=0$, the hypersurfaces $\hat{S}_{1}$ and $\hat{S}_{2}$ have their normal vectors equal to $\left(n_{10}, \ldots, n_{1 n}\right)$ and $\left(n_{20}, \ldots, n_{2 n}\right)$, respectively. Hence, by continuity, for small $t$, the hypersurface $\tilde{S}_{1}$ and $\hat{S}_{2}$ would be space-like with respect to $\tilde{u}_{1}$ and $\hat{u}_{1}$. Hence the values of $\tilde{u}_{1}$ and $\hat{u}_{1}$ in the domain $\tilde{G}_{1} \cap \hat{G}_{1}$ will depend only on the initial values in $G_{01}$ where $\tilde{u}_{1}(x, 0)=\hat{u}_{1}(x, 0)=u_{01}(x)$. Consequently, we have $\tilde{u}_{1}(x, t)=\hat{u}_{1}(x, t)$ in $G_{1}=\tilde{G}_{1} \cap \hat{G}_{1}$.

Together with the value $u_{1}(x, t)$ in $\tilde{G}_{1} \cap \hat{G}_{1}$, we also get the values of $\tilde{u}_{k}(x, t)$ and $\hat{u}_{2}(x, t)$ in $\tilde{G}_{k}$ and $\hat{G}_{2}$. In particular, we get the intersecting submanifold $Z$ of the hypersurfaces $\tilde{S}_{1}$ and $\hat{S}_{2}$.

Case (iii). This is the intermediate case between case (i) and case (ii). As in case (ii), we extend $S_{01}$ beyond $Z_{0}$ and extend $u_{01}, u_{0 k}$ into $\tilde{u}_{01}$ and $\tilde{u}_{0 k}$. By solving the corresponding Cauchy problem, we get the solution $\tilde{u}_{1}(x, t), \tilde{u}_{k}(x, t)$ and the separating shock front $\tilde{S}_{1}$, for small $t$.

By assumption (H7), as we have done in case (i), we can solve $q_{1}$ functions $\left(D p_{2}, \ldots, D p_{k}\right)$ and $\left(u_{2}, \ldots, u_{k-1}\right)$ from $q_{2}$ relations $Q(U)=0$. Thus we get a submanifold $Z$ of dimension $(n-1)$ on $\tilde{S}_{1}$.

Summing up, in all three cases in (1.6), we have constructed submanifold $Z$ on which are defined $q_{1}$ functions satisfying (1.7), (1.8) and (1.10). This concludes our first step in the proof of the Theorem. 
In our next step, we begin by constructing two smooth hypersurfaces $Y_{1}$ and $Y_{2}$ such that $Z=Y_{1} \cap Y_{2}$ and the following conditions are satisfied:

(1) $Y_{1}, Y_{2}$ are not tangent, at $t=0$, to any $S_{0 i}$ at $Z_{0}$;

(2) In case (i), $\left.Y_{1}\right|_{t=0} \subset G_{01},\left.Y_{2}\right|_{t=0} \subset G_{01}$ and $Y_{1}, Y_{2}$ are both space-like with respect to $u_{01}$. In fact, we will choose $Y_{1}$ near the direction $\left(n_{20}, \ldots, n_{2 n}\right)$ and $Y_{2}$ near the direction $\left(n_{10}, \ldots, n_{1 n}\right)$ in the neighborhood of $Z_{0}$. Since $\left(n_{20}, \ldots, n_{2 n}\right)$ and $\left(n_{10}, \ldots, n_{1 n}\right)$ are space-like with respect to $u_{01}$, so are the hypersurfaces $Y_{1}$ and $Y_{2}$.

In case (ii), $\left.Y_{1}\right|_{t=0} \subset G_{02},\left.Y_{2}\right|_{t=0} \subset G_{0 k}$. Also for $Y_{1}$ near $\left(n_{30}, \ldots, n_{3 n}\right), Y_{2}$ near $\left(n_{k 0}, \ldots, n_{k n}\right)$, they are space-like with respect to $u_{02}$ and $u_{0 k}$, respectively.

In case (iii), $\left.Y_{1}\right|_{t=0} \subset G_{01},\left.Y_{2}\right|_{t=0} \subset G_{0 k}$. And $Y_{1}, Y_{2}$ are space-like with respect to $u_{01}$ and $u_{0 k}$ respectively.

Having constructed the hypersurfaces $Y_{1}$ and $Y_{2}$, we are ready to perform the necessary transformation of variables.

First, we perform a transformation in $R^{n}$ such that in new coordinates, $Z_{0}=$ $\left\{x ; x_{1}=x_{2}=0\right\}$ and the projections of $Y_{1}$ and $Y_{2}$ onto the hyperplane $x_{1}=0$ are the domains $x_{2} \leq 0$ and $x_{2} \geq 0$, respectively. Let $Y_{2}=\left\{(t, x) ; t=r(x), x_{2} \geq\right.$ $0\}$. Extending $r(x)$ smoothly into $x_{2}<0$, denoted by $\tilde{r}(x)$, and performing the transformation

$$
x^{\prime}=x, \quad t^{\prime}=t-\tilde{r}(x),
$$

we get $Y_{2}=\left\{\left(t^{\prime}, x^{\prime}\right) ; t^{\prime}=0, x_{2}^{\prime} \geq 0\right\}$ in new coordinates. In order to simplify the notation, we will omit the prime in the following.

Now, the plane $t=0$ becomes $t+\tilde{r}(x)=0$. By another transformation, we can make this hypersurface become the hyperplane $x_{1}=0$, with the original domain $t>0$ becoming $x_{1}>0$. Notice that by all these transformations, the solvability of the problems remains equivalent and the space-like hypersurfaces remain to be space-like.

Let $Y_{1}=\left\{(t, x) ; t=s(x), x_{2} \leq 0\right\}$ in new coordinates. Let $r^{2}=t^{2}+x_{2}^{2}$, $y=\arctan \left(t / x_{2}\right)$, then $Y_{1}$ can be written as $y=K\left(r, x_{1}, x_{3}, \ldots, x_{n}\right)$ where $\pi / 2<$ $y<3 \pi / 2$. Now perform the transform

$$
\left\{\begin{array}{l}
t^{\prime}=r \sin (\pi / K) y, \\
x_{2}^{\prime}=r \cos (\pi / K) y, \\
x_{1}^{\prime}=x_{1}, x_{3}^{\prime}=x_{3}, \ldots, x_{n}^{\prime}=x_{n} .
\end{array}\right.
$$

Then, in the new coordinates, $Y_{1}$ is a half plane $\left\{t^{\prime}=0, x_{2}^{\prime}<0\right\}$, while $Y_{2}$ remains to be $\left\{t^{\prime}=0, x_{2}^{\prime}<0\right\}$.

REMARK 1. Here we notice that transformation (2.2) has singularity at $t=x_{2}=$ 0 when taken as a transform in the whole space, because it transforms a broken surface into a smooth one. But it is a diffeomorphism in any domain $y_{1}<y<y_{2}$, with $y_{2}-y_{1}<\pi$.

REMARK 2. If in particular, we can take $Y_{1}$ and $Y_{2}$ to be two parts of one smooth hypersurface $t=r(x)$, as in the physical examples we discuss in $\S 3$, then the transform changing $Y_{2}$ into $t=0$ automatically changes $Y_{1}$ into $t=0$. 
After all these transformations, we denote the resulted quasilinear hyperbolic system as

$$
\begin{aligned}
A_{0}(u) D_{t} u+A_{1}(u) D_{x_{1}} u+\sum_{2}^{n} A_{j}(u) D_{x_{j}} u+B(u) u=F(x, t), & \\
& \text { in } x_{1}>0, t>0 .
\end{aligned}
$$

Here we omit the prime in new coordinates.

On $x_{1}=0, t>0$, we have the boundary condition

$$
u=u_{0}\left(t, x^{\prime}\right) .
$$

Here $u_{0}\left(t, x^{\prime}\right)$ is in fact part of the $u_{0}$ in (1.3), after the transformation (2.2) and (2.3). It is worth pointing out that the hyperplane $x_{1}=0$ is space-like in new coordinates, though we call (2.5) a boundary condition.

On $t=0, x_{1}>0$, we impose the initial condition

$$
u(x, 0)=w_{0}(x)
$$

where the value of $w_{0}(x)$ is determined as follows.

In fact, in all three cases of (1.6), we can always extend $S_{01}, S_{02}$ and $u_{01}, u_{02}$, $u_{0 k}$ as we did before for the case (ii), and then construct the one shock wave solution separately. As in the case (ii), we denote by $\tilde{u}_{1}(x, t)$ and $\tilde{u}_{k}(x, t)$ the shock wave solution resulted from extending $S_{01}, u_{01}$ and $u_{0 k}$, denote by $\hat{u}_{1}(x, t)$ and $\hat{u}_{2}(x, t)$ the shock wave solution resulted from extending $S_{02}, u_{01}$ and $u_{02}$.

As we pointed out in case (ii), $\tilde{u}_{1}(x, t)=\hat{u}_{1}(x, t)$ in their common domain of definition, independent of the way of the extension. Also in the cases (i) and (iii), by the same argument, we have $\tilde{u}_{1}(x, t)=\hat{u}_{1}(x, t)$ in their common domain of definition.

Now, we take $w_{0}(x)$ as follows: If $\left.Y_{1}\right|_{t=0}$ (or $\left.Y_{2}\right|_{t=0}$ ) lies in $G_{01}, w_{0}(x)$ takes the value of $\tilde{u}_{1}(x, t)=\hat{u}_{1}(x, t)$ (transformed by (2.2) and (2.3)). If $\left.Y_{1}\right|_{t=0}\left(\left.Y_{2}\right|_{t=0}\right)$ lies in $G_{02}\left(G_{0 k}\right), w_{0}(x)$ takes the transformed value of $\tilde{u}_{2}(x, t)\left(\hat{u}_{k}(x, t)\right)$.

With $w_{0}(x)$ thus determined, we are to consider the initial-boundary value problem (2.4)-(2.6). Here the initial data $w_{0}(x)$ have jump discontinuity at $x_{2}=0$, the boundary $x_{1}=0$ is space-like and the boundary data $u_{0}\left(t, x^{\prime}\right)$ is piecewise smooth having jump discontinuity surfaces emitting from $x_{1}=x_{2}=0$.

From the assumptions (H2) and (H7), we know by implicit function theorem that for small $x_{1}>0$, there exist sufficiently smooth functions $\left(u_{i}, \lambda_{j}\right)$ on $x_{2}=0$ such that the transformed Rankine-Hugoniot conditions (1.5) are satisfied. Here $i=2, \ldots, k ; j=1, \ldots, k$ (for case (i)) or $i=3, \ldots,(k-1) ; j=3, \ldots, k$ (for case (ii)) or $i=2, \ldots,(k-1) ; j=2, \ldots, k$ (for case (iii)). Since the set of coefficients for which uniform stability conditions are satisfied is open, we know that for small $x_{1}>0$ and for these $\left(u_{i}, \lambda_{j}\right)$, the uniform stability conditions (H2) is satisfied. In particular, at $x_{1}=0, u_{i}$ 's are equal to the boundary value $u_{0}$ and $\lambda_{j}$ coincide with the tangent directions of the discontinuity surfaces of $u_{0}$.

The problem (2.4)-(2.6) is very much like the multishock wave problem we discussed in [1], except that we now have the boundary condition (2.5). 
As a matter of fact, for the multishock wave problem without boundary condition on $x_{1}=0$, we proved in [1] the following:

THEOREM FOR MULTISHOCK WAVES. For the Cauchy problem of the hyperbolic conservation laws (1.1) with initial data $u^{0}=\left(u_{+}^{0}, u_{-}^{0}\right)$ which have jump discontinuity along one smooth hypersurface $S_{0}$, if

(i) there are smooth functions $u_{i}^{0}, \lambda_{j}$ on $S_{0}, i=2, \ldots, k ; j=1, \ldots, k$; such that Rankine-Hugoniot conditions are satisfied for $k$ sets of $\left(\lambda_{j}, u_{j}^{0}, u_{j+1}^{0}\right), j=1, \ldots, k$; with $u_{1}^{0}=u_{-}^{0}, u_{k+1}^{0}=u_{+}^{0}$;

(ii) these $k$ shock fronts are separately linear stable in the sense of Majda in [4];

(iii) high order compatibility conditions are satisfied;

then, there exists a positive $t_{0}$, such that in $\left[0, t_{0}\right]$, there is a piecewise smooth solution of (1.1) with $k$ shock fronts.

Now for the problem (2.4)-(2.6), we can proceed just as in [1] in proving the above theorem. First of all, we perform the transformation containing the unknown shock fronts so that the original free boundary problem is reduced to a fixed multiboundary problem with newly introduced unknown functions $p_{j}$, describing the position of the shock fronts. Second, by the compatibility hypothesis (H5), we can construct an approximating solution $\left(u_{i}^{0}, p_{j}^{0}\right)$ such that the problem is further reduced to a problem for $\left(v_{i}, q_{j}\right)=\left(u_{i}^{0}-u_{i}, p_{j}^{0}-p_{j}\right)$, which satisfied the homogeneous initial and boundary conditions at $t=0$ and $x_{1}=0$, with the right side of the equation having zero traces at $t=0$ and $x_{1}=0$ up to sufficiently high order. Then let $t^{\prime}=\log t, x_{2}^{\prime}=x_{2} / t$, we get a new problem without initial conditions and all the boundaries are uncoupled. Such a problem can be treated similarly as in [1] and the existence of the solution follows.

This concludes our proof of the theorem.

3. Interaction of isentropic shock fronts in 2-dimensional space. As the application of our Theorem, we consider isentropic hydrodynamic equations of polytropic gas in 2-dimensional space:

$$
\left\{\begin{array}{l}
D_{t} f+D_{x_{1}}\left(f v_{1}\right)+D_{x_{2}}\left(f v_{2}\right)=0 \\
D_{t}\left(f v_{1}\right)+D_{x_{1}}\left(f v_{1}^{2}+p\right)+D_{x_{2}}\left(f v_{1} v_{2}\right)=0 \\
D_{t}\left(f v_{2}\right)+D_{x_{1}}\left(f v_{1} v_{2}\right)+D_{x_{2}}\left(f v_{2}^{2}+p\right)=0
\end{array}\right.
$$

Here $v_{1}, v_{2}$ are velocities of the gas in $x_{1}, x_{2}$ directions; $f$ is the density of the gas and $p=A f^{r}$ with $A, r$ constants and $r>1$.

The problem we are going to discuss comes from the interaction of two shock fronts, as G. Metivier discussed in [7] for $2 \times 2$ systems in 2-dimensional space. Here, for physical examples, (3.1) is a $3 \times 3$ system.

The problem of interaction of two shock fronts can be reduced to the problem in $\S 1$ with $k=4$, provided that only two shock fronts are produced after the interaction.

First, we consider the special case of constant state. Suppose at $t=0, u=$ $\left(f, v_{1}, v_{2}\right)$ is piecewise constant in $R^{n}$ with four discontinuity straight lines intersecting at $x_{1}=x_{2}=0$. See Figure 3 .

Denote the four discontinuity straightlines by $S_{ \pm 1}$ and $S_{ \pm 2}$, the four domains between them by $G_{1}, G_{ \pm}, G_{2}$, and the values of $u$ in four domains by $u_{1}, u_{ \pm}, u_{2}$. 


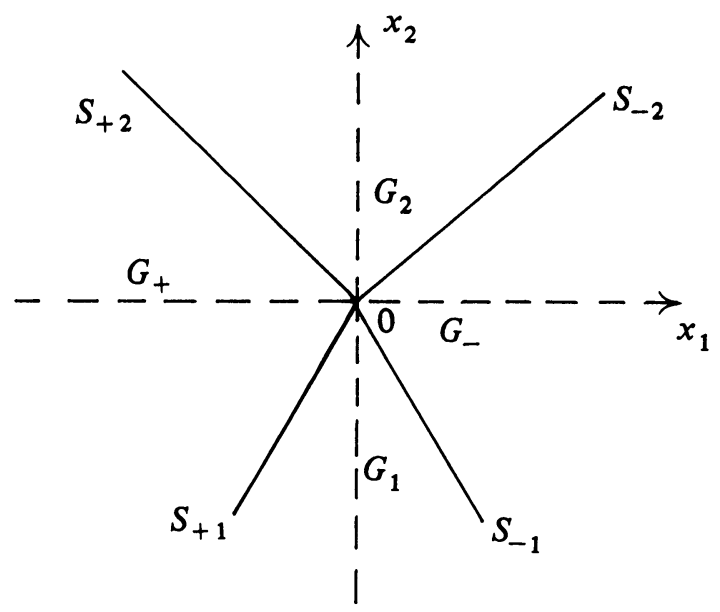

FIGURE 3

We will restrict ourselves to the discussion of the special case where the data are symmetric with respect to $x_{2}$ axis, i.e.

$$
\left\{\begin{array}{l}
S_{ \pm 1}=\left\{x ; x_{2}= \pm k_{1} x_{1}\right\} \\
S_{ \pm 2}=\left\{x ; x_{2}= \pm k_{2} x_{1}\right\}
\end{array} \quad k_{1}, k_{2}>0\right.
$$

and

$$
v_{+1}=-v_{-1}, \quad v_{+2}=v_{-2}=v_{2}, \quad v_{11}=v_{12}=v_{21}=0, \quad f_{+}=f_{-} .
$$

Since shock fronts are not characteristic, from Rankine-Hugoniot conditions it follows that the tangential speed to $S_{ \pm 1}$ in $G_{ \pm}$must be equal to the tangential speed in $G_{1}$, i.e., zero. So velocity $\left(v_{ \pm 1}, v_{ \pm 2}\right)$ in $G_{ \pm}$should satisfy

$$
v_{ \pm 1} \pm k_{1} v_{ \pm 2}=0
$$

Similarly, the tangential velocities on two sides of $S_{ \pm 2}$ being equal leads to

$$
v_{\mp 1} \pm k_{2} v_{\mp 2}=v_{21} \pm k_{2} v_{22}= \pm k_{2} v_{22} \text {. }
$$

Let the shock front direction of $S_{ \pm 1}$ be $\left(h_{ \pm 1}, \mp k_{1}, 1\right)$. Taking $h_{+1}=h_{-1}=h_{1}$ and by (3.3) and (3.4), we get from Rankine-Hugoniot condition:

$$
\left\{\begin{array}{l}
h_{1}\left(f_{+}-f_{1}\right)+k_{1}^{2} f_{+} v_{2}+f_{+} v_{2}=0 \\
-h_{1} f_{+} v_{2}-f_{+} v_{2}^{2}\left(1+k_{1}^{2}\right)-\left(p_{+}-p_{-}\right)=0 .
\end{array}\right.
$$

Similarly, let the shock front direction of $S_{ \pm 2}$ be $\left(h_{ \pm 2}, \pm k_{2}, 1\right)$. Taking $h_{+2}=$ $h_{-2}=h_{2}$ and noticing (3.3), (3.5), we get

$$
\left\{\begin{array}{l}
f_{+}\left(h_{2}+v_{2}+k_{2}^{2}\left(v_{2}-v_{22}\right)\right)-f_{2}\left(h_{2}+v_{22}\right)=0, \\
f_{+}\left(h_{2}+v_{2}+k_{2}^{2}\left(v_{2}-v_{22}\right)\right)\left(v_{2}-v_{22}\right)+\left(p_{+}-p_{2}\right)=0 .
\end{array}\right.
$$

The hypothesis $(\mathrm{H} 3)$ for $\left(h_{1}, \mp k_{1}, 1\right)$ and $\left(h_{2}, \pm k_{2}, 1\right)$ lying in a common plane becomes very explicit now. In 3-dimensional space, three vectors lie in a common plane if and only if the corresponding $3 \times 3$ matrix is degenerate. Thus we have

$$
\operatorname{det}\left|\begin{array}{rrr}
h_{1} & k_{1} & 1 \\
h_{1} & -k_{1} & 1 \\
h_{2} & k_{2} & 1
\end{array}\right|=0, \quad \operatorname{det}\left|\begin{array}{lrr}
h_{1} & k_{1} & 1 \\
h_{1} & -k_{1} & 1 \\
h_{2} & -k_{2} & 1
\end{array}\right|=0
$$


or equivalently

$$
h_{1}=h_{2} .
$$

Here, because $k_{1} \neq k_{2}$, so (3.8) does not mean that $S_{+1}$ and $S_{+2}$ have the same slope with respect to $t=0$.

In (3.6)-(3.8), we have five relations for nine variables $\left(h_{1}, h_{2}, f_{+}, f_{1}, f_{2}, v_{2}, v_{22}\right.$, $\left.k_{1}, k_{2}\right)$. We should choose these parameters such that (3.6)-(3.8) are satisfied and $h_{1}, h_{2}>0, f_{2}>f_{+}>f_{1}$. For the variables thus chosen, our hypotheses (H1)-(H3) are all satisfied.

Eliminating $h_{1}, h_{2}, k_{1}, k_{2}$ in (3.6)-(3.8), we get

$$
f_{2}\left(v_{2}-v_{22}\right)\left(A f_{1}^{r}-A f_{+}^{r}+f_{1} v_{2} v_{22}\right)+A\left(f_{+}^{r}-f_{2}^{r}\right) f_{1} v_{2}=0 .
$$

Now fixing $f_{+}=1$ and $f_{2}>1$, we denote the function on the left-hand side of (3.9) by $T\left(f_{1}\right)$,

$$
T\left(f_{1}\right)=f_{2}\left(v_{2}-v_{22}\right)\left(A f_{1}^{r}-A+f_{1} v_{2} v_{22}\right)+A\left(1-f_{2}^{r}\right) f_{1} v_{2} .
$$

For $v_{22}<v_{2}<0$, we have

$$
\left\{\begin{array}{l}
T(0)=f_{2}\left(v_{2}-v_{22}\right)(-A)<0 \\
T(1)=f_{2}\left(v_{2}-v_{22}\right) f_{1} v_{2} v_{22}+A\left(1-f_{2}^{r}\right) v_{2}>0 .
\end{array}\right.
$$

Consequently, there exists at least one $f_{1} \in(0,1)$ such that $(3.9)$ is satisfied and $0<f_{1}<f_{+}<f_{2}$.

Now for $\varepsilon<<1$, we take

$$
v_{2}=O\left(\varepsilon^{4}\right), \quad v_{22}=O\left(\varepsilon^{2}\right), \quad 1-f_{2}=O(\varepsilon) .
$$

Then from (3.6)-(3.8), we have

$$
\begin{aligned}
& h_{1}>0, \quad 1-f_{1}=O\left(\varepsilon^{3}\right), \quad h_{1}=O\left(\varepsilon^{-1}\right), \text { and } \\
& 1+k_{1}^{2}=\frac{A}{f_{1} v_{2}^{2}}\left(f_{1}-1\right)\left(f_{1}^{r}-1\right)=O\left(\varepsilon^{-2}\right) .
\end{aligned}
$$

Therefore, for $\varepsilon<<1$, we have a real positive solution for $k_{1}$.

From (3.7), we have

$$
k_{2}^{2}\left(v_{2}-v_{22}\right)=f_{2}\left(h_{1}+v_{22}\right)-\left(h_{1}+v_{2}\right)=\left(f_{2}-1\right) h_{1}+f_{2} v_{22}-v_{2} .
$$

Since $v_{2}-v_{22}>0,\left(f_{2}-1\right) h_{1}>0,\left(f_{2}-1\right) h_{1}=O(1), f_{2} v_{22}-v_{2}=O\left(\varepsilon^{2}\right)$, so (3.13) also has a real solution for $k_{2}$.

Thus, we can choose $\left(h_{1}, h_{2}, f_{1}, f_{+}, f_{2}, v_{2}, v_{22}, k_{1}, k_{2}\right)$ such that (3.6)-(3.8) are satisfied and $h_{1}, h_{2}>0, f_{2}>f_{+}>f_{1}$. Consequently (H1)-(H3) are satisfied.

(H4) is satisfied because $f_{+}=f_{-}>f_{1}$, and so $G_{1}$ is the required domain. (H5), (H6) are automatically satisfied for constant initial data. For variable data, (H5), (H6) are also satisfied if the data are $C^{\infty}$ tangent to the constant data at $x=0$.

Now consider (H7). For the problem with constant initial data, (H7) is not needed. For the general problem with variable initial data, noticing that our problem corresponds to case (ii) in (1.6), we have $k=4, q=k-3=1, m=3$. Therefore, $q=1<2=m-1$, contradictory to Corollary 1 in section 1 . Nevertheless, if we consider the special case of interaction of two symmetric shock fronts, so the Rankine-Hugoniot conditions on $S_{ \pm 2}$ are symmetric. We will have three 


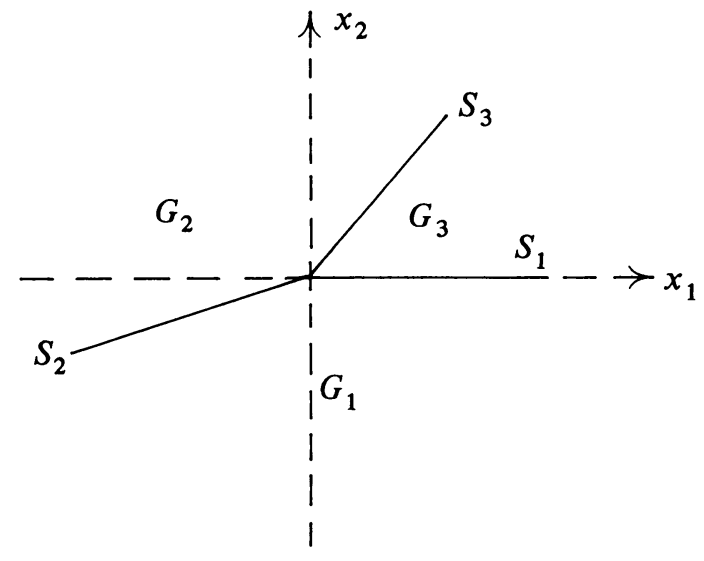

FIGURE 4

unknown functions $h_{2}, v_{2}, f_{2}$. Hence the condition in Corollary 1 is satisfied. Since (H7) is to exclude certain submanifold of lower dimension, by suitably choosing initial data, we can also make (H7) satisfied.

In summary, with the above chosen initial data, we get the local existence result for the problem of interaction of two symmetric isentropic shock fronts in 2-dimensional space, by the Theorem in $\S 1$.

4. An example of nonexistence. Again, we consider the isentropic hydrodynamic equations for polytropic gas (3.1). At $t=0, u=\left(f, v_{1}, v_{2}\right)$ is piecewise smooth in $R^{2}$ with three discontinuity straight lines intersecting at $x_{1}=x_{2}=0$. See Figure 4.

Without loss of generality, we assume $S_{1}=\left\{x ; x_{2}=0, x_{1}>0\right\}, S_{2}=\left\{x ; x_{1}=\right.$ $\left.k_{2} x_{2}, x_{2}<0\right\}, S_{3}=\left\{x ; x_{1}=k_{3} x_{2}, x_{2}>0\right\}$, and $v_{11}=v_{12}=0$. Let $\left(h_{1}, 0,1\right)$, $\left(h_{2},-1, k_{2}\right),\left(h_{3},-1, k_{3}\right)$ be the shock front directions on $S_{1}, S_{2}$ and $S_{3}$, then from the fact that the tangential speeds on two sides of a shock front should be equal, i.e.,

$$
v_{31}=0, \quad k_{2} v_{21}+v_{22}=0, \quad v_{32}=k_{3} v_{21}+v_{22}=\left(k_{3}-k_{2}\right) v_{21}=0
$$

we deduce from Rankine-Hugoniot conditions

$$
\begin{gathered}
\left\{\begin{array}{l}
h_{1}\left(f_{3}-f_{1}\right)+f_{3} v_{32}=h_{1}\left(f_{3}-f_{1}\right)+\left(k_{3}-k_{2}\right) v_{21}=0, \\
h_{1} f_{3} v_{32}+f_{3} v_{32}^{2}+p_{3}-p_{1}=0 .
\end{array}\right. \\
\left\{\begin{array}{l}
h_{2}\left(f_{2}-f_{1}\right)-f_{2} v_{21}\left(1+k_{2}^{2}\right)=0, \\
h_{2} f_{2} v_{21}-f_{2} v_{21}^{2}\left(1+k_{2}^{2}\right)-\left(p_{2}-p_{1}\right)=0 .
\end{array}\right. \\
\left\{\begin{array}{c}
h_{3}\left(f_{3}-f_{2}\right)+v_{21}\left(f_{2}+f_{2} k_{2} k_{3}-f_{3} k_{2} k_{3}+f_{3} k_{3}^{2}\right)=0, \\
-h_{3} f_{2} v_{21}+\left(1+k_{2} k_{3}\right) f_{2} v_{21}^{2}-\left(p_{3}-p_{2}\right)=0 .
\end{array}\right.
\end{gathered}
$$

In order to have three stable shock fronts intersecting on a common line, (4.2)(4.4) must be satisfied together with the condition for $\left(h_{1}, 0,1\right),\left(h_{2},-1, k_{2}\right)$ and $\left(h_{3},-1, k_{3}\right)$ lying in a common plane

$$
\operatorname{det}\left|\begin{array}{rrr}
h_{1} & 0 & 1 \\
h_{2} & -1 & k_{2} \\
h_{3} & -1 & k_{3}
\end{array}\right|=h_{1}\left(k_{2}-k_{3}\right)-\left(h_{2}-h_{3}\right)=0
$$


We are going to show that there is no set of data $\left(v_{21}, f_{1}, f_{2}, f_{3}, k_{2}, k_{3}, h_{1}, h_{2}, h_{3}\right)$ satisfying (4.2)-(4.5) with $f_{3}>f_{2}>f_{1}$ for a convex state function $p=p(f)$.

Eliminating $h_{1}, h_{2}, h_{3}$ from (4.2)-(4.5), we get

$$
\left\{\begin{array}{l}
\left(f_{3} v_{21}\right)^{2}\left(k_{3}-k_{2}\right)^{2}=\left(f_{3}-f_{1}\right)\left(p_{3}-p_{1}\right) \\
f_{1} f_{2} v_{21}^{2}\left(1+k_{2}^{2}\right)=\left(f_{2}-f_{1}\right)\left(p_{2}-p_{1}\right) \\
f_{2} f_{3} v_{21}^{2}\left(1+k_{3}^{2}\right)=\left(f_{3}-f_{2}\right)\left(p_{3}-p_{2}\right) \\
\frac{\left(k_{2}-k_{3}\right)^{2} f_{3}}{f_{3}-f_{1}}-\frac{\left(1+k_{2}^{2}\right) f_{2}}{f_{2}-f_{1}}-\frac{f_{2}+f_{3} k_{3}^{2}}{f_{3}-f_{2}}+k_{2} k_{3}=0
\end{array}\right.
$$

Further eliminating $v_{21}^{2}$ and $k_{2}, k_{3}$ from (4.6), we have the relation for $\left(f_{1}, f_{2}, f_{3}\right)$, which after simplification can be written as

$$
f_{2} f_{3}\left(p_{2}-p_{3}\right)+f_{1} f_{3}\left(p_{3}-p_{1}\right)-f_{2} f_{1}\left(p_{2}-p_{1}\right)=0 \text {. }
$$

Fixing $f_{1}, f_{2}$, we write the left-hand side of $(4.7)$ as $M\left(f_{3}\right)$. Since $M\left(f_{2}\right)=0$, we need only to show that $M^{\prime}\left(f_{3}\right)<0$ for $f_{3}>f_{2}$.

$$
\begin{aligned}
M^{\prime}\left(f_{3}\right) & =f_{2} p\left(f_{2}\right)-f_{1} p\left(f_{1}\right)+\left(f_{1}-f_{2}\right) p\left(f_{3}\right)+\left(f_{1}-f_{2}\right) f_{3} p^{\prime}\left(f_{3}\right) \\
& =\left(f_{1}-f_{2}\right)\left(f_{3} p^{\prime}\left(f_{3}\right)+p\left(f_{3}\right)\right)+\left(f_{2}-f_{1}\right)\left(f^{*} p^{\prime}\left(f^{*}\right)+p\left(f^{*}\right)\right)
\end{aligned}
$$

where $f^{*}=f_{1}+\theta\left(f_{2}-f_{1}\right), 0<\theta<1$.

From $f_{3}>f^{*}, p^{\prime}>0, p^{\prime \prime}>0$, we get $f^{*} p^{\prime}\left(f^{*}\right)+p\left(f^{*}\right)<f_{3} p^{\prime}\left(f_{3}\right)+p\left(f_{3}\right)$, consequently $M^{\prime}\left(f_{3}\right)<0$. Thus we know (4.7) is never satisfied for $f_{3}>f_{2}$. Therefore we get the conclusion that there can be no such shock fronts in $R^{2} \times R_{+}$ for hydrodynamic gas equations with convex state function, which have three stable shock fronts intersecting at a common curve.

ACKNOWLEDGment. I would like to thank Professor A. T. Bui for the encouragement and the valuable discussions with him while preparing this paper. This work was supported by NSERC of Canada.

\section{REFERENCES}

1. A. T. Bui and D. Li, Double shock fronts for hyperbolic conservation laws in several space variables, preprint.

2. H-O Kreiss, Initial boundary value problems for hyperbolic systems, Comm. Pure Appl. Math. 23 (1970), 277-298.

3. D. $\mathrm{Li}$, The shock wave solutions of quasilinear hyperbolic-parabolic coupled systems and the linear stability of isothermal radiative shock waves in multi-dimensional space, Sci. Sinica (Ser. A) 30 (1987), 1-16.

4. A. Majda, The stability of multi-dimensional shock fronts, Mem. Amer. Math. Soc. No. 275, 1983.

5. __ The existence of multi-dimensional shock fronts, Mem. Amer. Math. Soc. No. 281, 1983.

6. G. Metivier, Interaction de chocs, Seminaire Bony-Sjostrand-Meyer, 1984-1985.

7. __ Interaction de deux chocs pour un systeme de deux lois de conservation, en dimension deux d'espace, Trans. Amer. Math. Soc. 296 (1986), 431-479.

8. J. Smoller, Shock wave and reaction-diffusion equations, Springer-Verlag, 1983.

Department of Mathematics, University of British Columbia, Vancouver V6T 1W5, BRITISH COlUmbia, CaNADa

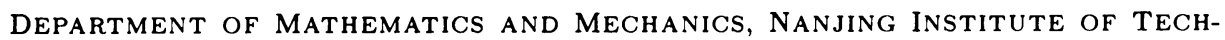
Nology, Nanjing, PEOPle's Republic of China

Current address: Department of Mathematics, University of Colorado, Boulder, Colorado 80309 